\title{
LncRNA PLAC 2 Is Downregulated in Osteosarcoma and Regulates Cancer Cell Proliferation Through miR-93
}

This article was published in the following Dove Press journal: Cancer Management and Research

\section{Xiangran Sun \\ Ling Yu \\ Yubo Shi \\ Weichun Guo}

Department of Orthopedics, Renmin Hospital of Wuhan University, Wuhan 430060, Hubei Province, People's

Republic of China
Correspondence: Weichun Guo Department of Orthopedics, Renmin Hospital of Wuhan University, Hubei Zhang Road Wuchang District No. 99 Jiefang Road 238, Wuhan 430060, Hubei Province, People's Republic of China

Tel +86-27-8804I9|I-82209

Fax +86-27-8804I9II

Email pj264I@I63.com
Introduction: PLAC 2 is a tumor-suppressive lncRNA in glioma, while its roles in other types of cancer remain unclear. This study was carried out to explore the potential involvement of PLAC 2 in osteosarcoma (OS).

Methods: Expression levels of PLAC 2 in OS and paired non-tumor tissues from OS patients were determined by RT-qPCR. A follow-up study was performed to analyze the prognostic value of PLAC 2 for OS. Interactions between PLAC 2 and miR-93 were assessed by cell transfection, followed by RT-qPCR. Cell proliferation assay was performed to analyze cell proliferation.

Results: Our results showed that PLAC 2 was downregulated in OS tissues, and the high expression levels of PLAC 2 were associated with favorable overall survival of OS patients. MiR-93 was upregulated in OS tissues and its expression was inversely correlated with the expression of PLAC 2. In OS cells, overexpression of PLAC 2 resulted in downregulated miR-93, while overexpression of miR-93 did not affect the expression of PLAC 2. Overexpression of PLAC 2 led to decreased proliferation rate of OS cells, while overexpression of miR-93 showed opposite roles and reduced the overexpressing effects of PLAC 2.

Conclusion: PLAC 2 is downregulated in OS and regulates cancer cell proliferation through miR-93.

Keywords: osteosarcoma, PLAC 2, miR-93, survival, proliferation

\section{Background}

Osteosarcoma (OS) is a primary malignant tumor originates from the skeleton and is characterized by the formation of osteoid tissue or immature bone by cancer cells. ${ }^{1}$ Most cases of OS develop from long bones, and cases originate from soft tissues are rare. ${ }^{2}$ OS mostly affects teenagers and young adults between 10 and 25 years old. ${ }^{2}$ However, OS may also affect elderly and the prognosis is generally poor. ${ }^{3}$ Surgical resection of the primary tumors is the only radical treatment for early stage OS, ${ }^{4}$ while surgery is not applicable for advanced stage OS. ${ }^{4}$ The application of chemotherapies and radiotherapies may improve the short-term survival, while long-term prognosis is still not satisfactory. ${ }^{5}$ Therefore, novel therapies are still needed.

Unclear pathogenesis is the major challenge in the development of novel antiOS therapies, ${ }^{6,7}$ such as targeted therapies, ${ }^{8}$ which aim to interfere with OS-related gene expression. ${ }^{8}$ Besides genes involved in protein synthesis, the occurrence and 
progression of OS also involves non-coding RNAs (ncRNAs), ${ }^{9}$ which participate in biological processes by regulating gene expression. ${ }^{10}$ It has been reported that the regulation of certain critical ncRNAs involved in the pathogenesis of OS may contribute to the treatment of OS. ${ }^{11}$ In a recent study, PLAC 2 was characterized as a tumor-suppressive long ( $>200 \mathrm{nt})$ ncRNA (lncRNA) in glioma. ${ }^{12}$ Our preliminary RNA-seq analysis revealed the downregulation of PLAC 2 in OS and its inverse correlation with miR-93, which can promote OS cell proliferation. ${ }^{13}$ This study aimed to investigate the interactions between miR-93 and PLAC 2 in OS.

\section{Methods}

\section{OS Patients and Tissue Collection}

This study passed the review of the Ethics Committee of Renmin Hospital of Wuhan University. This study enrolled 68 OS patients ( 36 females and 32 males, 15 to 26 years old, mean age $20.6 \pm 2.1$ years old) who were admitted to aforementioned hospital between May 2012 and June 2014. All patients had no previous history of malignancies. Only newly diagnosed OS cases were included. OS patients complicated with other clinical disorders and/or had been treated before admission were excluded from this study. All patients or their guardians signed the informed consent. Fine needle biopsies were performed on all OS patients to collect both OS tumor and paired non-tumor (within $5 \mathrm{~cm}$ around tumors) tissue samples. All samples were subjected to histopathological examinations to confirm the correct samples were obtained. All fresh samples were stored in liquid nitrogen.

\section{Treatment and a 5-Year Follow-Up}

The determination of therapies was performed based on AJCC staging system. According to this system and clinical findings, the 68 OS patients included 21, 17, 18 and 12 cases at stage I, II, III and IV, respectively. A follow-up study on the 68 patients was performed for 5 years or until their deaths to record their survival conditions. All 68 patients completed the follow-up.

\section{Cells and Cell Culture}

Human OS cell lines MG-63 and U2OS were obtained from ATCC (USA). Cells were cultivated in a medium composed of 10\% FBS and 90\% EMEM medium. Cells were cultivated under conditions of $5 \% \mathrm{CO}_{2}, 95 \%$ humidity and $37^{\circ} \mathrm{C}$. Cells were harvested at $80 \%$ confluence to perform the following experiments.

\section{Cell Transfections}

PLAC 2 expression vector was constructed using pcDNA3.1 vector (Invitrogen) as the backbone. MiR-93 mimic and negative control (NC) miRNA were purchased from Sigma-Aldrich (USA). MG-63 and U2OS cells were transfected with $40 \mathrm{mM}$ miRNA or $10 \mathrm{mM}$ vector using lipofectamine 2000 reagent (Sangon biotech). Cells without transfections were control (C) cells. Cells transfected with NC miRNA or empty vector were negative control (NC) cells. The following experiments were performed at $24 \mathrm{~h}$ post-transfection.

\section{RNA Preparations and RT-qPCR Assays}

Total RNA Purification Kit (Cat. 17200, Norgen biotek) was used to extract total RNAs from in vitro cultivated cells and two types of tissues. PureLink miRNA Isolation Kit (Thermo Fisher Scientific) was used to extract miRNAs from aforementioned cells and tissues. All RNA samples were digested with gDNA eraser (Takara) to remove genomic DNAs. QuantiTect Reverse Transcription Kit (QIAGEN) was used to prepare cDNA samples through reverse transcriptions (RTs) with total RNA samples as template. All qPCR reaction mixtures were prepared using DyNAmo Flash SYBR Green qPCR Kit (Thermo Fisher Scientific) with GAPDH as endogenous control to measure the expression levels of PLAC 2. The expression levels of mature miR-93 were measured using All-in-One ${ }^{\mathrm{TM}}$ miRNA qRT-PCR Reagent Kit (Genecopoeia) with all steps performed following the manufacturer's instructions. All PCR reactions were performed in triplicate manner and fold changes of gene expression levels were analyzed by $2^{-\Delta \Delta \mathrm{CT}}$ method.

\section{CCK-8 Assay}

The effects of transfections on the proliferation of MG-63 and U2OS cells were evaluated by CCK- 8 assay using Cell Counting Kit 8 (ab228554, Abcam). Cells were seeded onto a 96-well cell culture plate (3000 cells per well), followed by cell culture under aforementioned conditions. CCK-8 solution was added into each well of a 96-well cell culture plate to reach the final concentration of $10 \%$ at $4 \mathrm{~h}$ before collecting cells. Cells were collected at 24, 48, 72 and 96 $\mathrm{h}$ and $\mathrm{OD}$ values were measured at $450 \mathrm{~nm}$.

\section{Statistical Analysis}

All experiments were performed in 3 independent biological replicates and data were expressed as mean \pm standard 
deviation (SD). Paired $t$ test was used to compare differences between OS and non-tumor tissues. ANOVA (one-way) and Tukey's test were used to compare differences among multiple groups. The 68 patients were grouped into high and low PLAC 2 level groups $(n=34)$ and survival curves were plotted using GraphPad Prism6 software and compared by Log-rank test. Pearson's correlation coefficient was used to analyze correlations. $P<0.05$ was statistically significant.

\section{Results}

\section{PLAC 2 Was Downregulated in OS and Predicted Poor Survival}

Expression levels of PLAC 2 in paired non-tumor and OS tissue samples from the 68 OS patients were measured by qPCR assay. Paired $t$ test analysis showed that the expression levels of PLAC 2 were significantly lower in OS tissues in comparison to that in non-tumor tissues (Figure 1A, $p<0.001)$. Survival curves were plotted for both high and low PLAC 2 level groups and compared. It was observed that the overall survival rate of patients in low-level group was lower than that of patients in high-level group (Figure 1B).

\section{MiR-93 Was Upregulated in OS Tissues and Inversely Correlated with PLAC 2}

Expression levels of miR-93 in the paired non-tumor and OS tissue samples from the 68 OS patients were also measured by qPCR assay. Paired $t$ test analysis showed that the expression levels of miR-93 were significantly higher in OS tissues in comparison to that in non-tumor tissues (Figure 2A, $p<0.001$ ). Correlation analysis showed that the expression levels of miR-93 and PLAC 2 were inversely and significantly correlated across OS tissue samples (Figure 2B). In contrast, the correlation between miR-93 and PLAC 2 was not significant across non-tumor tissue samples (Figure 2C).

\section{Overexpression of PLAC 2 Led to Downregulated miR-93 in MG-63 and U2OS Cells}

MG-63 and U2OS cells were transfected with miR-93 mimic or PLAC 2 expression vector to assess the interactions between PLAC 2 and miR-93. Compared to the $\mathrm{C}$ and NC groups, the expression levels of PLAC 2 and miR-93 were significantly increased at $24 \mathrm{~h}$ post-transfection (Figure 3A, $p<0.05$ ). In addition, compared to the $\mathrm{C}$ and $\mathrm{NC}$ groups, overexpression of PLAC 2 led to downregulation of miR-93 (Figure 3B, $p<0.05$ ). In contrast, overexpression of miR-93 did not affect the expression of PLAC 2 (Figure 3C).

\section{Overexpression of PLAC 2 Suppressed the Proliferation of MG-63 and U2OS Cells Through miR-93}

The effects of overexpression of PLAC 2 and miR-93 on the proliferation of MG-63 and U2OS cells were evaluated by CCK-8 assay. Compared to the $\mathrm{C}$ group, overexpression of

B

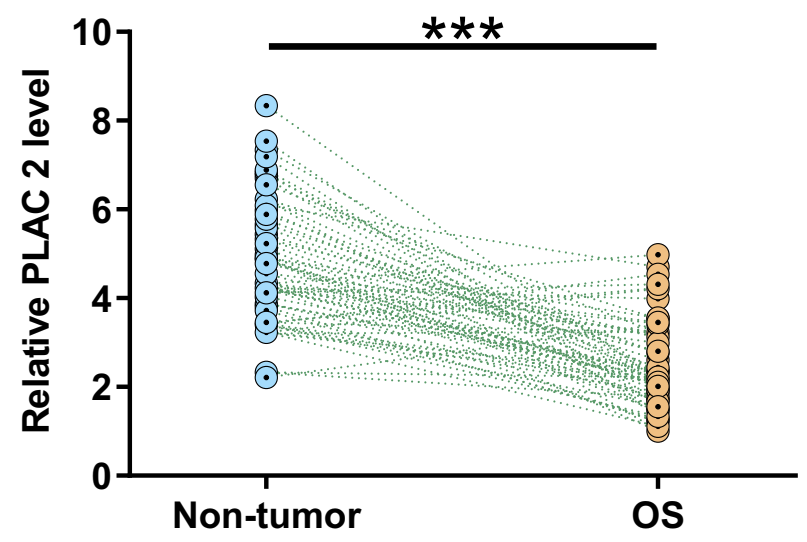

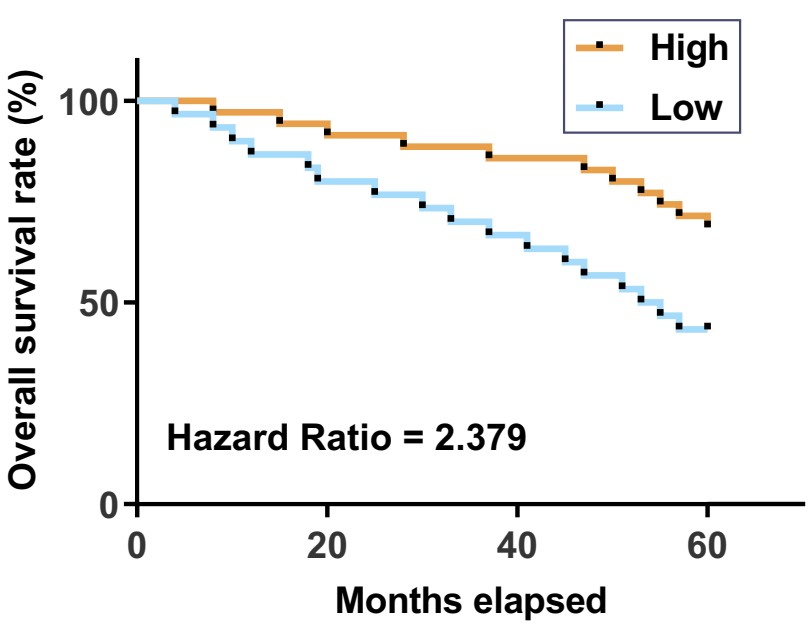

Figure I PLAC 2 was downregulated in OS and predicted poor survival. Expression levels of PLAC 2 in the 68 pairs of non-tumor and OS tissue samples from the 68 OS patients were measured by performing qPCR assay. Paired $t$ test was used to compare the mean values from 3 biological replicates $(\mathbf{A})$. $* * * p<0.00 \mathrm{I}$. The 68 patients were grouped into high and low PLAC 2 level groups $(n=34$, cutoff value $=3.5 \mathrm{I})$. Survival curves were plotted using GraphPad Prism6 software based on the 5-year follow-up data and compared by Log rank test (B). 
A

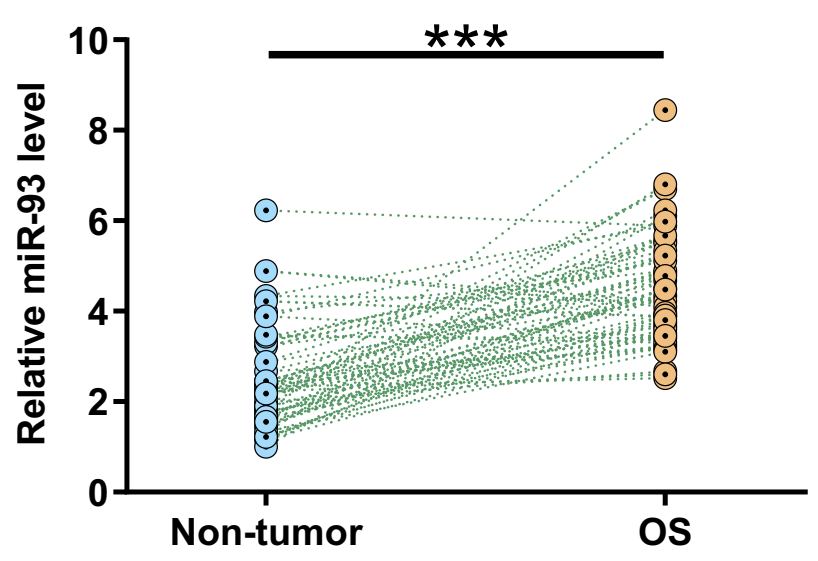

C

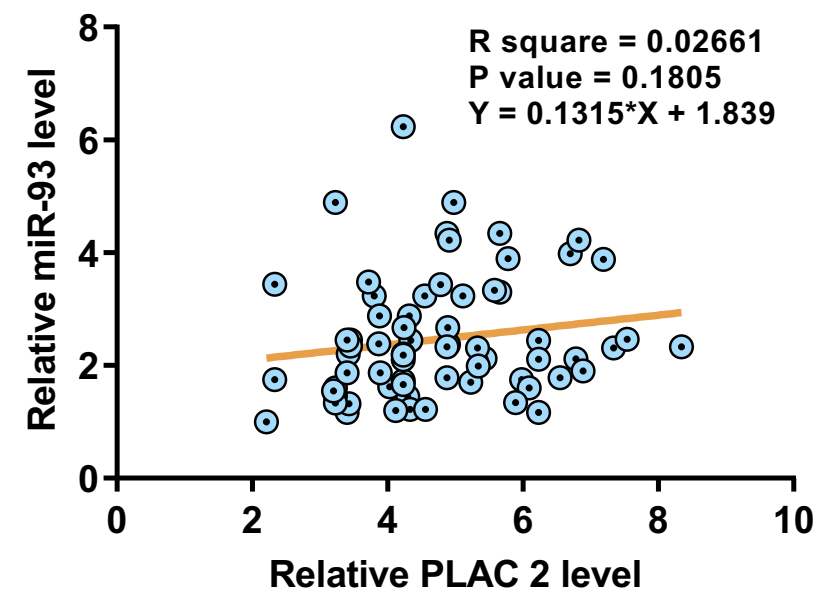

B

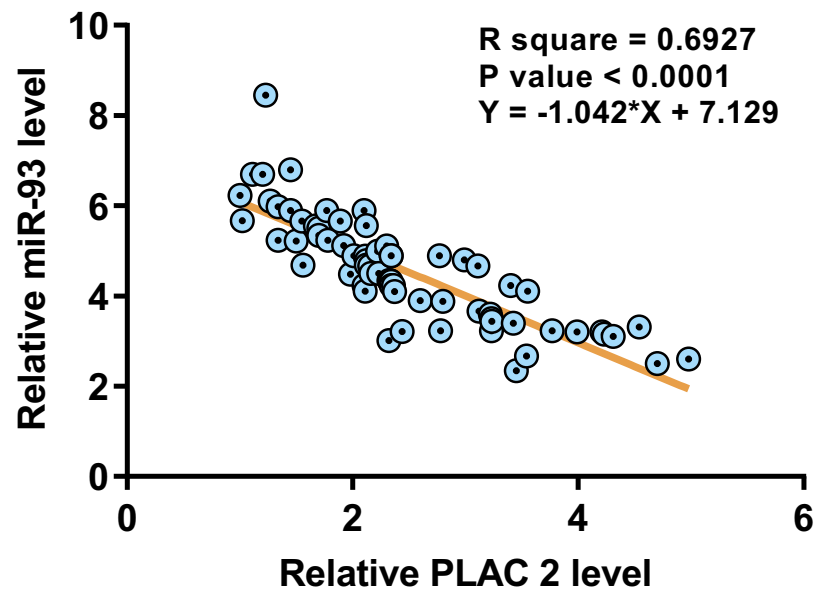

Figure 2 MiR-93 was upregulated in OS tissues and inversely correlated with PLAC 2. Expression levels of miR-93 in the 68 pairs of non-tumor and OS tissue samples from the 68 OS patients were also measured by performing qPCR assay. Paired $t$ test was used to compare the mean values from 3 biological replicates (A). $* * * p<0.00 \mathrm{I}$. Pearson's correlation coefficient was used to analyze correlations between expression levels of miR-93 and PLAC 2 across OS (B) and non-tumor (C) tissue samples.

PLAC 2 caused decreased proliferation rate of OS cells, while overexpression of miR-93 showed opposite roles and reduced the effects of overexpressing PLAC 2 (Figure 4, $p<0.05)$.

\section{Discussion}

In this study, we mainly investigated the expression pattern and functionality of PLAC 2 in OS. We observed the downregulation of PLAC 2 in OS and its inhibitory effects on miR-93, which has been characterized as an oncogenic miRNA in OS.

A recent study reported that PLAC 2 was strongly downregulated in glioma, and it interacted with STAT1 to downregulate RPL36 and suppress cancer cell cycle transition to inhibit cancer development. ${ }^{12}$ Several other studies have also reported the involvement of PLAC 2 in other types of cancer. For instance, PLAC 2 is downregulated in non-small cell lung cancer and suppresses cancer development by downregulating miR-21. ${ }^{14}$ PLAC 2 upregulates p53 in hepatocellular carcinoma to induce cell apoptosis. ${ }^{15}$ In contrast, PLAC 2 activates Wnt/ $\beta$-catenin pathway to promote cell proliferation in oral squamous cell carcinoma. ${ }^{16}$ Therefore, PLAC 2 may play different roles in different cancers. To the best of your knowledge, our study is the first to report the downregulation of PLAC 2 in OS. In addition, we also observed the inhibited cell proliferation after the overexpression of PLAC 2 . Therefore, PLAC 2 is likely a tumor suppressor in OS.

With the advantage of novel therapeutic approaches, the survival of OS has been significantly improved during 
A
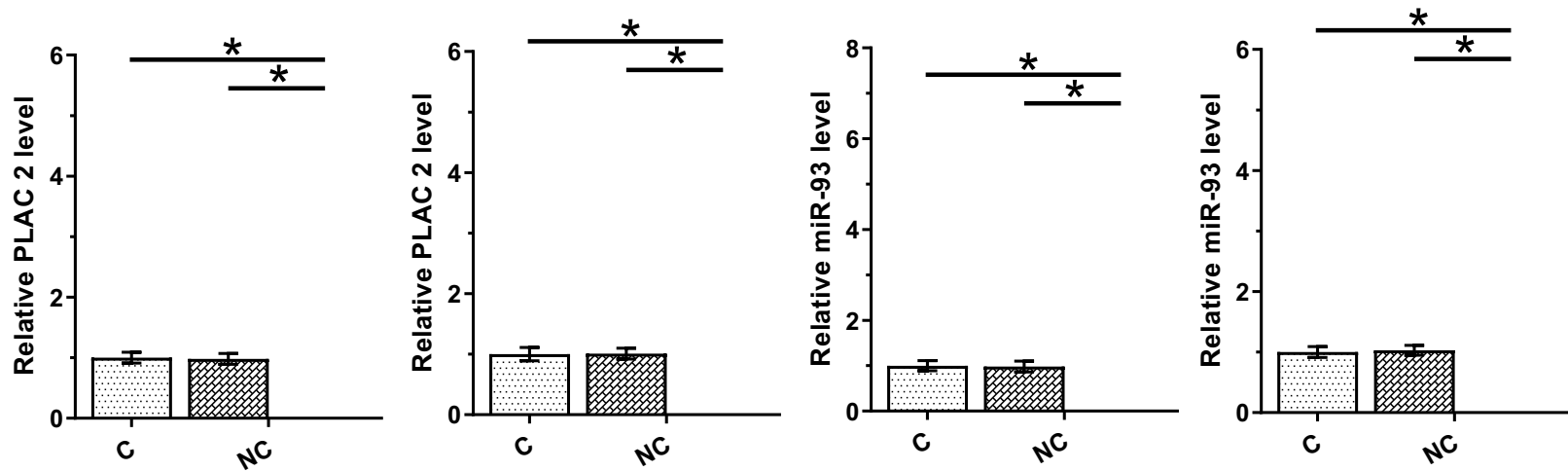

B
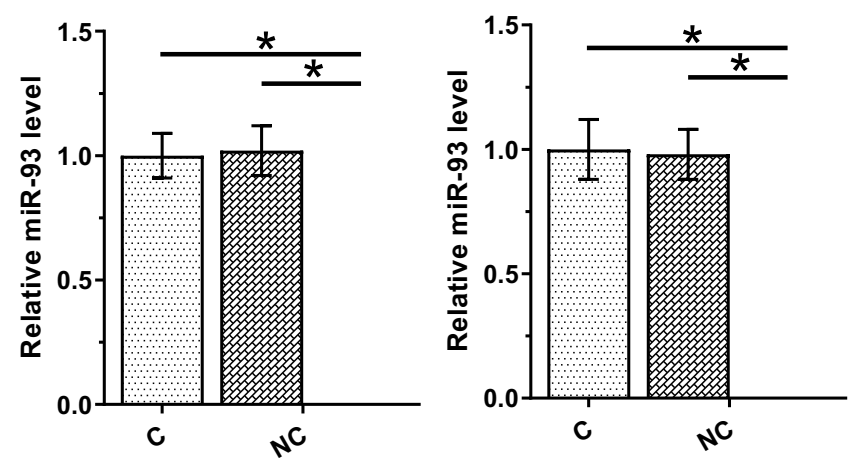

C
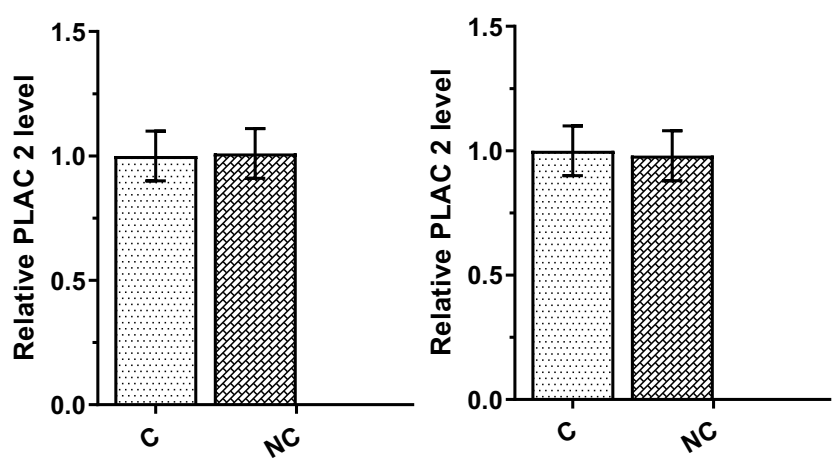

Figure 3 Overexpression of PLAC 2 led to downregulated miR-93 in MG-63 and U2OS cells. MG-63 and U2OS cells were transfected with miR-93 mimic or PLAC 2 expression vector to analyze the interactions between PLAC 2 and miR-93. Overexpression of PLAC 2 and miR-93 was confirmed at $24 \mathrm{~h}$ post-transfection (A). The effects of overexpression of PLAC 2 on miR-93 (B) and the effects of overexpression of miR-93 on PLAC 2 (C) were also analyzed by qPCR at 24 h post-transfection. ANOVA (one-way) and Tukey's test were used to compare the mean values from 3 biological replicates (A). C, control cells without transfection; NC, negative control cells transfected with empty vector or NC miRNA; *p $<0.05$.

A

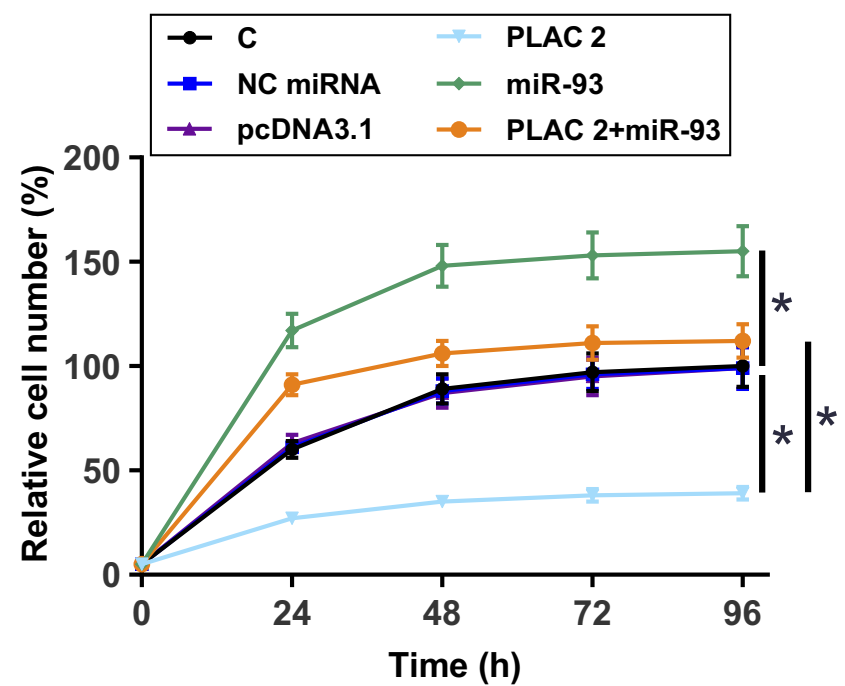

B

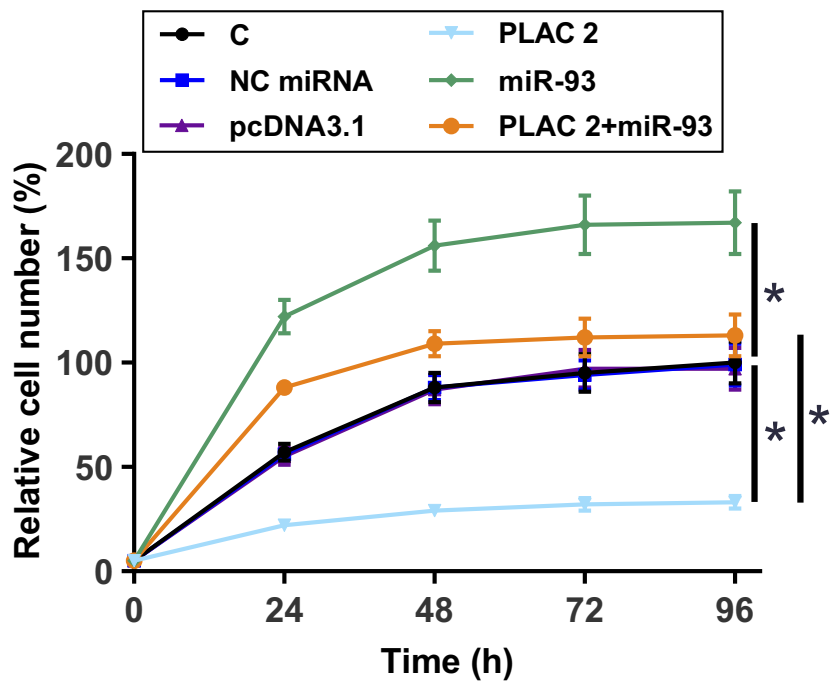

Figure 4 Overexpression of PLAC 2 suppressed the proliferation of MG-63 and U2OS cells through miR-93. The effects of overexpressing PLAC 2 and miR-93 on the proliferation of MG-63 and U2OS cells were analyzed by CCK-8 assay. ANOVA (one-way) (A) and Tukey's test were used to compare the mean values from 3 biological replicates (B). C, control cells without transfection; ${ }^{*} p<0.05$. 
the past decades. ${ }^{17,18}$ However, death is inevitable in some cases, especially in advanced OS cases. In this study, we demonstrated that the low expression levels of PLAC 2 were significantly correlated with the poor survival of OS patients. Therefore, measurement of the expression levels of PLAC 2 may assist the prognosis of OS, thereby help the determination of treatment approaches and development of post-operative care program. However, the prognostic accuracy remains to be further evaluated by future studies with much bigger sample size.

MiR-93 has been proven as an oncogenic miRNA in many types of cancer, such as OS and prostate cancer. ${ }^{13,19}$ In OS, miR-93 can target PTEN to inhibit the proliferation of cancer cells. ${ }^{13}$ In this study, we observed the upregulation of miR-93 in OS and the increased proliferation rate of OS cells after the overexpression of miR-93. Therefore, our data confirmed the oncogenic roles of miR-93 in OS. Interestingly, our results showed that PLAC 2 could downregulate miR-93, while the mechanism is still unclear. However, we only observed the inverse correlation between PLAC 2 and miR-93 among OS tissue samples but not in non-tumor samples. Therefore, the interactions between PLAC 2 and miR-93 may be mediated by certain OS-related pathological factors.

In conclusion, PLAC 2 is downregulated in OS and its overexpression can downregulate miR-93 to suppress OS cell proliferation.

\section{Abbreviations}

OS, osteosarcoma; ncRNAs, non-coding RNAs; lncRNA, long (>200nt) ncRNA.

\section{Data Sharing Statement}

The analyzed data sets generated during the study are available from the corresponding author on reasonable request.

\section{Ethics and Consent Statement}

The present study was approved by the Ethics Committee of Renmin Hospital of Wuhan University. The research has been carried out in accordance with the World Medical Association Declaration of Helsinki. All patients or their guardians signed informed consent. A parent or legal guardian provided written informed consent for any patient under the age of 18 years.

\section{Author Contributions}

All authors made substantial contributions to conception and design, acquisition of data, or analysis and interpretation of data; took part in drafting the article or revising it critically for important intellectual content; gave final approval of the version to be published; and agree to be accountable for all aspects of the work.

\section{Disclosure}

The authors declare that they have no competing interests.

\section{References}

1. Lindsey BA, Markel JE, Kleinerman ES. Osteosarcoma overview. Rheumatol Ther. 2017;4(1):25-43. doi:10.1007/s40744-016-00 50-2

2. Picci P. Osteosarcoma (osteogenic sarcoma). Orphanet $J$ Rare Dis. 2007;2(1):6. doi:10.1186/1750-1172-2-6

3. Imura Y, Takenaka S, Kakunaga S, et al. Survival analysis of elderly patients with osteosarcoma. Int Orthop. 2019;43(7):1741-1747. doi:10.1007/s00264-019-04332-y

4. Isakoff MS, Bielack SS, Meltzer P, et al. Osteosarcoma: current treatment and a collaborative pathway to success. J Clin Oncol. 2015;33(27):3029-3035. doi:10.1200/JCO.2014.59.4895

5. Anderson ME. Update on survival in osteosarcoma. Orthop Clin North Am. 2016;47(1):283-292. doi:10.1016/j.ocl.2015.08.022

6. Gianferante DM, Mirabello L, Savage SA. Germline and somatic genetics of osteosarcoma - connecting aetiology, biology and therapy. Nat Rev Endocrinol. 2017;13(8):480-491. doi:10.1038/ nrendo.2017.16

7. Denduluri SK, Wang Z, Yan Z, et al. Molecular pathogenesis and therapeutic strategies of human osteosarcoma. J Biomed Res. 2016;30 (1):5.

8. Abarrategi A, Tornin J, Martinez-Cruzado L, et al. Osteosarcoma: cells-of-origin, cancer stem cells, and targeted therapies. Stem Cells Int. 2016;2016:3631764. doi:10.1155/2016/3631764

9. Chen R, Wang G, Zheng Y, et al. Long non-coding RNAs in osteosarcoma. Oncotarget. 2017;8(12):20462-20475. doi:10.18632/ oncotarget.14726

10. Mercer TR, Dinger ME, Mattick JS. Long non-coding RNAs: insights into functions. Nat Rev Genet. 2009;10(3):155-159. doi: $10.1038 / \mathrm{nrg} 2521$

11. Zhang Q, Geng PL, Yin P, et al. Down-regulation of long non-coding RNA TUG1 inhibits osteosarcoma cell proliferation and promotes apoptosis. Asian Pac J Cancer Prev. 2013;14(4):2311-2315. doi:10.7314/APJCP.2013.14.4.2311

12. Hu YW, Kang CM, Zhao JJ, et al. LncRNA PLAC 2 down-regulates RPL 36 expression and blocks cell cycle progression in glioma through a mechanism involving STAT 1. J Cell Mol Med. 2018;22 (1):497-510. doi:10.1111/jcmm.13338

13. Kawano M, Tanaka K, Itonaga I, et al. microRNA-93 promotes cell proliferation via targeting of PTEN in Osteosarcoma cells. J Exp Clin Cancer Res. 2015;34(1):76. doi:10.1186/s13046-015-0192-z

14. Xia H, Xiu M, Gao J, et al. LncRNA PLAC 2 downregulated miR-21 in non-small cell lung cancer and predicted survival. BMC Pulm Med. 2019;19(1):172. doi:10.1186/s12890-019-0931-6

15. Zheng Y, Lv P, Wang S, et al. LncRNA PLAC2 upregulates p53 to induce hepatocellular carcinoma cell apoptosis. Gene. 2019;712:143944. doi:10.1016/j.gene.2019.143944

16. Chen F, Qi S, Zhang X, et al. IncRNA PLAC2 activated by $\mathrm{H} 3 \mathrm{~K} 27$ acetylation promotes cell proliferation and invasion via the activation of $\mathrm{Wnt} / \beta$-catenin pathway in oral squamous cell carcinoma. Int J Oncol. 2019;54(4):1183-1194. doi:10.3892/ ijo.2019.4707 
17. Durfee RA, Mohammed M, Luu HH. Review of osteosarcoma and current management. Rheumatol Ther. 2016;3(2):221-243. doi:10.1007/ s40744-016-0046-y

18. Spraker-Perlman HL, Barkauskas DA, Krailo MD, et al. Factors influencing survival after recurrence in osteosarcoma: a report from the Children's Oncology Group. Pediatr Blood Cancer. 2019;66(1): e27444. doi:10.1002/pbc. 27444
19. Yang K, Li YW, Gao ZY, et al. MiR-93 functions as a tumor promoter in prostate cancer by targeting disabled homolog 2 (DAB2) and an antitumor polysaccharide from green tea (Camellia sinensis) on their expression. Int J Biol Macromol. 2019;125:557-565. doi:10.1016/j.ijbiomac.2018.12.088

\section{Publish your work in this journal}

Cancer Management and Research is an international, peer-reviewed open access journal focusing on cancer research and the optimal use of preventative and integrated treatment interventions to achieve improved outcomes, enhanced survival and quality of life for the cancer patient.
The manuscript management system is completely online and includes a very quick and fair peer-review system, which is all easy to use. Visit http://www.dovepress.com/testimonials.php to read real quotes from published authors. 